American Journal of Environmental Sciences 3 (1): 37-42, 2007

ISSN 1553-345X

(C) 2007 Science Publications

\title{
Reservoir Oscillations with Through Flow
}

\author{
A. A. Khan \\ 218 Lowry Hall, Department of Civil Engineering, Clemson University \\ Clemson, SC, 29634-0911, USA
}

\begin{abstract}
The two-reservoir problem with through flow is a common feature in wastewater treatment plants. The start of the through flow may cause water surface oscillations in the reservoirs and velocity variations in the connecting pipe during the initial unsteady phase that may adversely affect the operation and under certain conditions may cause overtopping of the upstream reservoir. The classical solution based on the rigid mass theory is inapplicable as velocity variations within the pipe system are ignored. One-dimensional mass and momentum conservation equations, based on the elastic theory, with a new set of boundary conditions are solved using the method of characteristics to investigate the water surface oscillations in the two reservoirs and minimum velocity in the connecting pipe as a result of the start of a through flow. The impact of a constant or variable friction factor on the velocity and water surface oscillations in the connecting pipe is found to be negligible. The magnitude and the time it takes to establish the through flow are found to be important parameters for the system. Optimum time of linear increase of a given inflow rate to the final steady state discharge is investigated for an actual facility. A ramp time of 40 seconds is found to reduce the maximum water surface level from $40 \mathrm{~cm}$ to $9.4 \mathrm{~cm}$ and increase the minimum velocity in the system to $85 \%$ of the final steady state velocity.
\end{abstract}

Keywords: Mathematical modeling, hydraulics and hydrodynamics, pipe flow

\section{INTRODUCTION}

Wastewater treatment facilities are commonly built with multiple reservoirs that are connected through pipes. The fluid with waste material is continuously moved from one reservoir to another with prescribed inflow at the upstream reservoir and outflow from the downstream reservoir. As the system is started from rest the flow takes sometime to reach a steady state. During the initial unsteady phase, oscillations in the water surface levels in the two reservoirs and flow velocity in the pipe occur. The velocity oscillations cause very low and sometimes negative velocity that may cause deposition of the waste material in the connecting pipe. The oscillations in the water surface levels may adversely affect the operation and under certain conditions may cause overtopping of the upstream reservoir. The classical solution of the water surface oscillations in a two-reservoir system ${ }^{[3]}$, which is based on the rigid mass theory, is not valid for predicting oscillation pattern in the two reservoirs with through flow. Therefore, the full mass and momentum conservation equations based on the elastic properties of the fluid and pipe must be solved numerically.

In this study, the mass and momentum conservation equations are solved using the method of characteristics. Appropriate boundary conditions for the upstream and downstream reservoirs are devised to obtain solution for the two-reservoir problem with through flow. The method is applied to predict the unsteady flow pattern in a real water treatment plant due to the start of a through flow. The results of water surface oscillation in the upstream and downstream reservoirs, the maximum and minimum velocities in the pipe, and the time it takes for the system to reach steady state are discussed. A method that can be easily adopted to reduce the amplitude of the water surface oscillations and increase the minimum velocity in the system is discussed.

Rigid mass theory: The water surface oscillations pattern in a two-reservoir system resulting from an initial perturbation of the water surface level has been solved traditionally using the mass and momentum equations. The mass and momentum equations used for this analysis are derived assuming the mass in the connecting pipe acts as a solid body. This means the whole mass is assumed to move as a single unit with a single velocity throughout the fluid system. The pipe friction acts as a damping factor that reduces the amplitude of the velocity and water surface oscillations with time. The situation is depicted in Fig. 1. The equations used to predict the oscillations of the water surface and velocity in a two-reservoir system can be found in any fluid mechanics book ${ }^{[3]}$ and are given below

$Z_{1} A_{1}=Z_{2} A_{2}=Z A$

Corresponding Author: A. A. Khan, 218 Lowry Hall, Department of Civil Engineering, Clemson, SC 29634-0911, USA, Tel: 864-656 3327, Fax: 864-656 2670 
$\frac{d V}{d t}+\frac{f L_{e}}{2 D L} V|V|+\frac{g A}{L}\left(1 / A_{1}+1 / A_{2}\right) Z=0$

$\frac{d Z}{d t}=V$

where $t$ is time, $Z_{1}$ and $Z_{2}$ are the water surface elevations in the two reservoirs with respect to the equilibrium position, $A_{1}$ and $A_{2}$ are the surface areas of the two reservoirs, $Z$ and $A$ are the water surface elevation and area in the connecting pipe, $f$ is the friction factor, $L$ is the length of the pipe, $L_{e}$ is the equivalent length of the pipe, i.e., length of the pipe plus additional length to account for minor losses, and $V$ is the velocity of the water mass in the pipe.

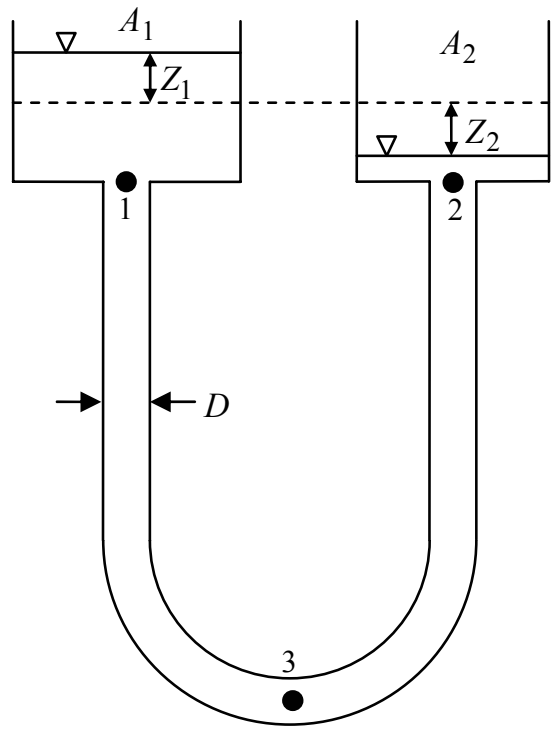

Fig. 1: Schematic of water level oscillations in two reservoirs

Equations (2) and (3) can be solved for $Z$ and $V$ using any numerical scheme, e.g. fourth order RungeKutta method. Equation (1) is then used to determine the corresponding change in water surface level in the two reservoirs. The solution of these equations is oscillatory in nature with pipe friction providing damping.

In this study, a two-reservoir problem is revisited with through flow that occurs in most water treatment plants. The mass and momentum conservation equations are used to model oscillations pattern that sets up due to the start of a through flow. In addition, the velocity oscillations pattern within the connecting pipe is determined that will provide flow development time within the pipe.

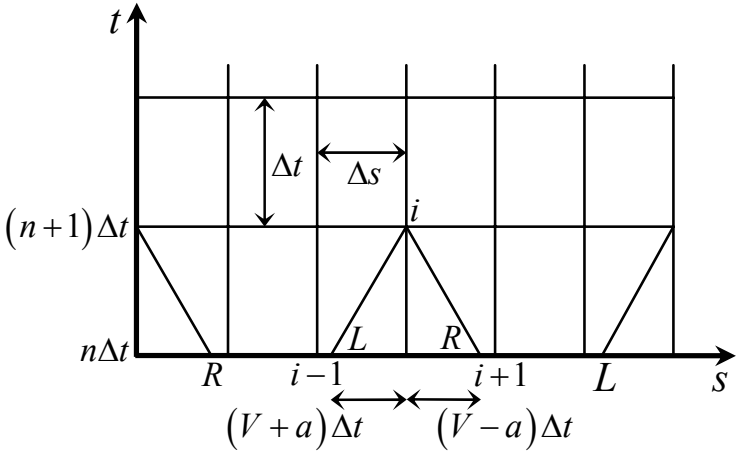

Fig. 2: Rectangular grid for the method of characteristics

Mass and momentum conservation equations: The mass and momentum conservation equations based on the elastic properties of the fluid and pipe, as given by Streeter et al. ${ }^{[4]}$, are given below

$$
\begin{aligned}
& \frac{1}{\rho} \frac{\partial p}{\partial t}+\frac{1}{\rho} \frac{\partial p}{\partial s}+a^{2} \frac{\partial V}{\partial s}=0 \\
& \frac{\partial V}{\partial t}+V \frac{\partial V}{\partial s}+\frac{1}{\rho} \frac{\partial p}{\partial s}+g \frac{\partial z}{\partial s}+\frac{f}{2 D} V|V|=0
\end{aligned}
$$

where $s$ is the distance along the pipe; $\rho$ is the density of the fluid; $p$ is the pressure; $z$ is the pipe elevation; and $a$ is the wave propagation speed that depends on the properties of the fluid (density and bulk modulus of elasticity), pipe (modulus of elasticity, pipe wall thickness, and diameter), and the way the pipe is anchored $^{[2]}$. The mass and momentum equations describe the variation of velocity and pressure along the whole length of the pipe. These equations can be solved using the method of characteristics. The discretized form of the positive and negative characteristic equations, based on equations described above, can be easily obtained (see for example Watters ${ }^{[5]}$ ) and are given below

$$
\begin{aligned}
& \frac{V_{i}^{n+1}-V_{L}}{\Delta t}+\frac{g}{a} \frac{H_{i}^{n+1}-H_{L}}{\Delta s}-\frac{g}{a} V_{L} \frac{d z}{d s}+ \\
& \frac{f}{2 D} V_{L}\left|V_{L}\right|=0 ; \quad \text { for } \quad \frac{d s}{d t}=V+a
\end{aligned}
$$

$\frac{V_{i}^{n+1}-V_{R}}{\Delta t}-\frac{g}{a} \frac{H_{i}^{n+1}-H_{R}}{\Delta s}+\frac{g}{a} V_{R} \frac{d z}{d s}+$ $\frac{f}{2 D} V_{R}\left|V_{R}\right|=0 ; \quad$ for $\quad \frac{d s}{d t}=V-a$

where $H$ represents the piezometric head or the elevation of the hydraulic grade line, $\Delta s$ is the size of the spatial discretization, and $\Delta t$ is the size of the 
temporal discretization. The two equations described above have two unknowns, velocity and piezometric head, at time $(n+1) \Delta t$ and can be determined explicitly from the values at the previous time step, $n \Delta t$, as shown in Fig. 2. The equations for determining the velocity and piezometric head at the new time level for all the interior nodes are given below

$$
\begin{gathered}
V_{i}^{n+1}=\frac{1}{2}\left[\begin{array}{l}
\left(V_{L}+V_{R}\right)+\frac{g}{a}\left(H_{L}-H_{R}\right)+ \\
\frac{g}{a} \Delta t\left(V_{L}-V_{R}\right) \frac{d z}{d s}- \\
\frac{\Delta t}{2 D}\left(f_{L} V_{L}\left|V_{L}\right|+f_{R} V_{R}\left|V_{R}\right|\right)
\end{array}\right] \\
H_{i}^{n+1}=\frac{1}{2}\left[\begin{array}{l}
\left(H_{L}+H_{R}\right)+\frac{a}{g}\left(V_{L}-V_{R}\right)+ \\
\Delta t\left(V_{L}+V_{R}\right) \frac{d z}{d s}- \\
\frac{a}{g} \frac{\Delta t}{2 D}\left(f_{L} V_{L}\left|V_{L}\right|-f_{R} V_{R}\left|V_{R}\right|\right)
\end{array}\right]
\end{gathered}
$$

where

$$
\begin{aligned}
& V_{L}=\frac{V_{i}^{n}+a\left(V_{i-1}^{n}-V_{i}^{n}\right) \Delta t / \Delta s}{1-\left(V_{i-1}^{n}-V_{i}^{n}\right) \Delta t / \Delta s} \\
& H_{L}=H_{i}^{n}+\left(H_{i-1}^{n}-H_{i}^{n}\right)\left(a+V_{L}\right) \Delta t / \Delta s \\
& V_{R}=\frac{V_{i}^{n}+a\left(V_{i+1}^{n}-V_{i}^{n}\right) \Delta t / \Delta s}{1+\left(V_{i+1}^{n}-V_{i}^{n}\right) \Delta t / \Delta s} \\
& H_{R}=H_{i}^{n}+\left(H_{i+1}^{n}-H_{i}^{n}\right)\left(a-V_{R}\right) \Delta t / \Delta s
\end{aligned}
$$

The friction factor, $f$, can be modified to include the minor losses within a computational cell. The modified friction factor is applied only to the side where a minor loss exists. If $K$ is the minor loss coefficient, the modified friction factor is given by

$$
f^{\prime}=f+K \frac{D}{\Delta s}
$$

The friction factors for the left and right cells are calculated based on the average velocity within these cells at the previous time step.

To calculate the velocity for the prescribed piezometric heads at the upstream and downstream ends, that is at nodes 1 and $N$, the following two equations are used, respectively

$$
\begin{aligned}
& V_{1}^{n+1}=\left[\begin{array}{l}
V_{R}+\frac{g}{a}\left(H_{1}^{n+1}-H_{R}\right)-\frac{g}{a} \frac{d z}{d s} V_{R} \Delta t- \\
\frac{f_{R} \Delta t}{2 D} V_{R}\left|V_{R}\right|
\end{array}\right] \\
& V_{N}^{n+1}=\left[\begin{array}{l}
V_{L}-\frac{g}{a}\left(H_{N}^{n+1}-H_{R}\right)+\frac{g}{a} \frac{d z}{d s} V_{L} \Delta t- \\
\frac{f_{L} \Delta t}{2 D} V_{L}\left|V_{L}\right|
\end{array}\right]
\end{aligned}
$$

The values of $H_{R}, V_{R}, H_{L}$, and $V_{L}$ are calculated using the equations given above. The piezometric heads at nodes 1 and $N$ are calculated from the reservoir elevations at the end of the previous time step. The water surface elevations of the two reservoirs at the end of each time step are calculated as follows

$$
Z_{1}^{n+1}=Z_{1}^{n}+\frac{1}{A_{1}}\left[Q_{i n} \Delta t-0.5 \Delta t\left(V_{1}^{n+1}+V_{1}^{n}\right) A\right]
$$

$$
Z_{2}^{n+1}=Z_{2}^{n}+\frac{1}{A_{2}}\left[0.5 \Delta t\left(V_{N}^{n+1}+V_{N}^{n}\right)-Q_{o u t} \Delta t\right]
$$

where $Q_{i n}$ is the inflow rate into the upstream reservoir and $Q_{o u t}$ is the outflow rate from the downstream reservoir. The outflow rate is calculated based on the water surface level at the previous time step in the downstream reservoir.

To simulate the classical problem of oscillations of water levels in the two reservoirs, the inflow and outflow in the above equations are ignored. To simulate the case of through flow, the inflow rate and a function relating the outflow rate to the water surface elevation in the downstream reservoir must be known. The inflow discharge, which can be constant or time varying, is usually controlled and known. The outflow from the reservoir can be controlled by weir or pumped at a known rate. In this study, it is assumed that the inflow is steady and known and the outflow is over a sharp crested weir. A sharp crested weir formula that relates discharge to the head over the weir is used with a coefficient of discharge of 0.6 .

\section{Classical Two Reservoir Oscillations Problem}

The aim of this test is to compare the solution of the classical method with the full mass and momentum equations, which are solved using the method of characteristics. For this test, the upstream reservoir is 20 square meters and the downstream reservoir is 30 square meters. The two reservoirs are connected by a 
700-m long, 0.6-m internal diameter, 1.5-cm thick PVC pipe. The friction factor is assumed constant throughout the simulation at 0.015 , however, the variation of friction factor with Reynolds number can be easily incorporated in the solution procedure. The value of the wave speed, $a$, is calculated to be about $288 \mathrm{~m} / \mathrm{s}$. The water level in the upstream reservoir is perturbed $15.0 \mathrm{~m}$ above the equilibrium position. The resulting solutions from the classical method and the method of characteristics, with wave speed of $288 \mathrm{~m} / \mathrm{s}$, $400 \mathrm{~m} / \mathrm{s}$, and $1232 \mathrm{~m} / \mathrm{s}$, are shown in Fig. 3. The results

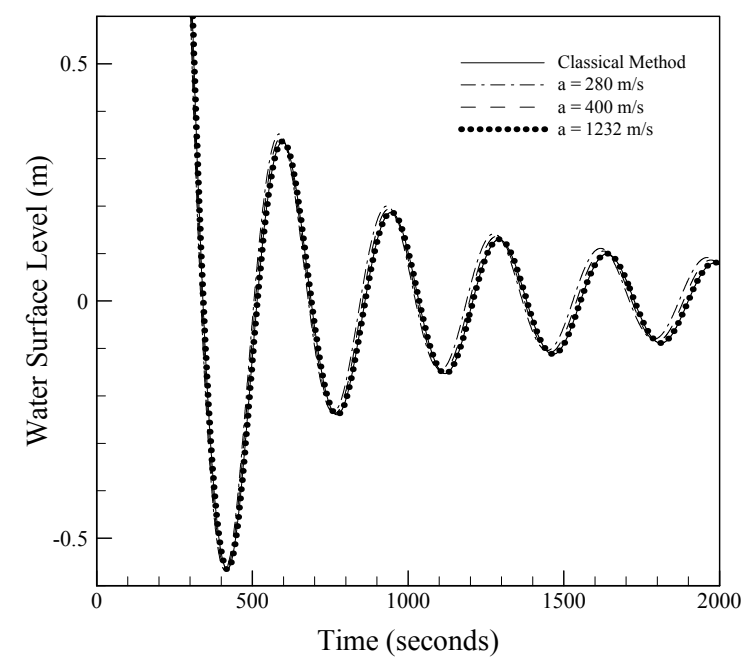

Fig. 3: Water surface variation in the upstream reservoir

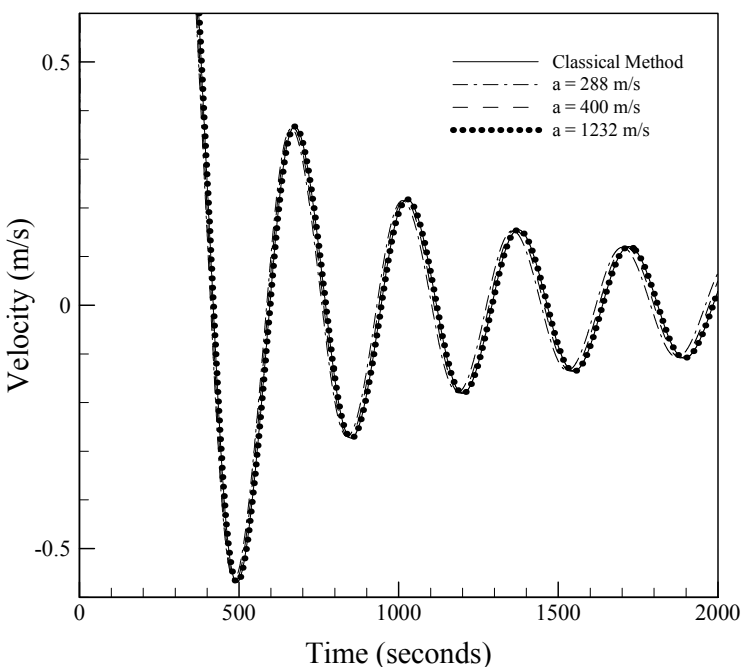

Fig. 4: Velocity variation in the pipe at the center of the pipe

show that as the wave speed increases the solution from the elastic theory approaches that of the classical theory. The wave speed of $1232 \mathrm{~m} / \mathrm{s}$ is for a pipe fabricated of steel. All the wave speeds are calculated assuming the pipe is rigidly anchored with no axial strain. Fig. 3 shows that the classical solution is an approximation of the full elastic theory with a large wave speed. For small wave speed, there is a phase shift as well as amplitude increase in the water surface oscillations compared to the classical solution. The velocity variations with time obtained from the two methods for various wave speeds at the middle of pipe (point 3 in Fig. 1) are shown in Fig. 4. For the classical method the whole water mass within the pipe is moving with the same velocity, whereas in the case of the method of characteristics, the velocity variation with time shown in Fig. 4 is at the center of the pipe. For small wave speed, the velocity oscillations are out of phase compared to the solution obtained from the classical method, however the amplitude compares quite well.

\section{Reservoir Oscillations Due to Through Flow}

Next, the full mass and momentum equations are solved to determine the oscillations pattern of the water surfaces in the reservoirs and the velocity in the pipe due to the start of a through flow. The example selected is based on the set up of an actual water treatment plant with an upstream reservoir area of 2.42 $\mathrm{m}^{2}$ and a downstream reservoir area of $965 \mathrm{~m}^{2}$. Both reservoirs are circular in shape. The length of the cast iron pipe connecting the two reservoirs is $42.7 \mathrm{~m}$, with pipe diameter of $76.2 \mathrm{~cm}$ (2.5 feet), and wall thickness of $22 \mathrm{~mm}$. The wave speed through the pipe, assuming rigidly anchored pipe with no axial strain, is calculated to be $3990 \mathrm{~m} / \mathrm{s}$. The design flow through the system is 710 cubic meters per hour (4.5 millions gallons per day). The friction factor during the solution process is calculated based on the steady flow condition using the explicit equation given by Haaland ${ }^{[1]}$. The equation is valid for the Reynolds number between 40000 and $10^{8}$. For the above pipe size, the minimum velocity for which this equation can be used is about $5 \mathrm{~mm} / \mathrm{s}$ and the Reynolds number for final steady state velocity (maximum velocity in the pipe) is $3.1 \times 10^{5}$, which is well below the maximum Reynolds number. The friction factor based on the final steady state velocity is 0.0167 .

The outflow from the downstream reservoir is over a sharp crested weir extending over the whole circumference of the reservoir. However, other types of outflows, such as a limited length of weir, spillway, or water pumped out at constant rate, etc., can be easily incorporated. Simulations are performed for the above scenario using a variable friction factor (i.e., friction factor for each cell is calculated based on the average velocity in the cell) and a constant friction factor of 0.0167 throughout the development period. Fig. 5 shows the variation of the computed water surface oscillations in the upstream reservoir for a constant and variable friction. The results show that the oscillations 
of water surface level have slightly higher amplitude in the case of variable friction. The variable friction has no impact on the wavelength of the oscillations. The computed velocity variation at the middle of the connecting pipe shows similar trend. The results show that the impact of a variable friction is negligible.

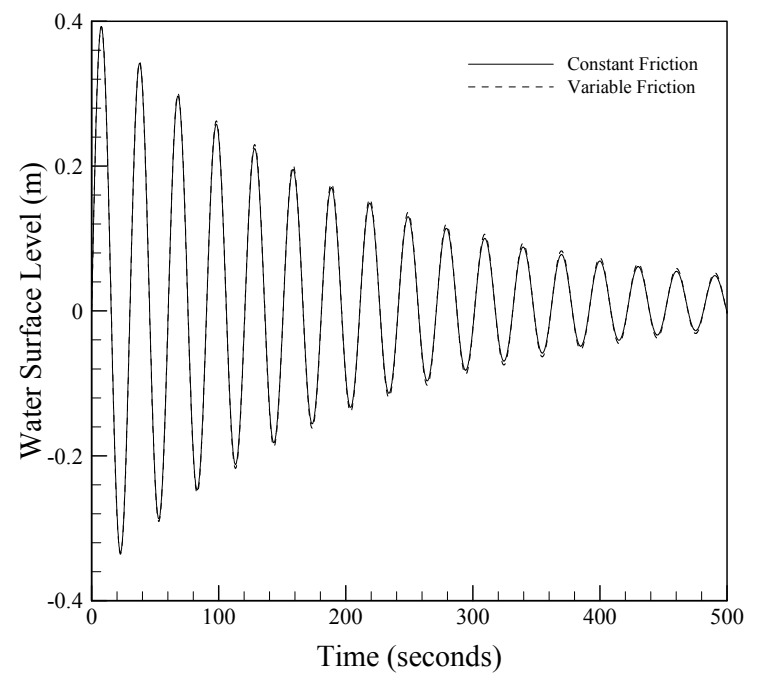

Fig. 5: Effect of friction on water surface oscillations in the upstream reservoir

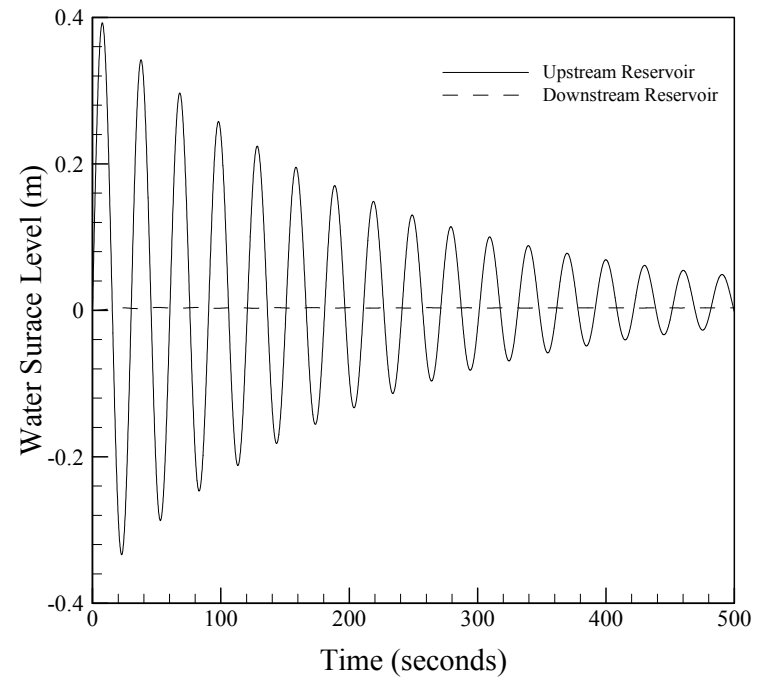

Fig. 6: Water surface oscillation in upstream and downstream reservoirs

Fig. 6 shows the variation of the computed water surface level in the upstream and downstream reservoirs. Due to a larger surface area of the downstream reservoir and outflow over the weir, the oscillations in the downstream reservoir are almost negligible when compared to the upstream reservoir. In the upstream reservoir, the maximum water surface level is $0.39 \mathrm{~m}$ above the initial level and the minimum level is $-0.33 \mathrm{~m}$ below the initial level. The final stabilized water level is $0.012 \mathrm{~m}$ above the initial water level. These values compare very well with the field observations. The computed velocity variations with time at the middle of the pipe are shown in Fig. 7. The maximum velocity is $0.83 \mathrm{~m} / \mathrm{s}$, the minimum velocity after the first peak is $0.059 \mathrm{~m} / \mathrm{s}$, and the final steady state velocity is $0.432 \mathrm{~m} / \mathrm{s}$. As the maximum velocity will mobilize solids in the flow, a very low velocity following a peak velocity may cause sudden deposition and operational difficulties.

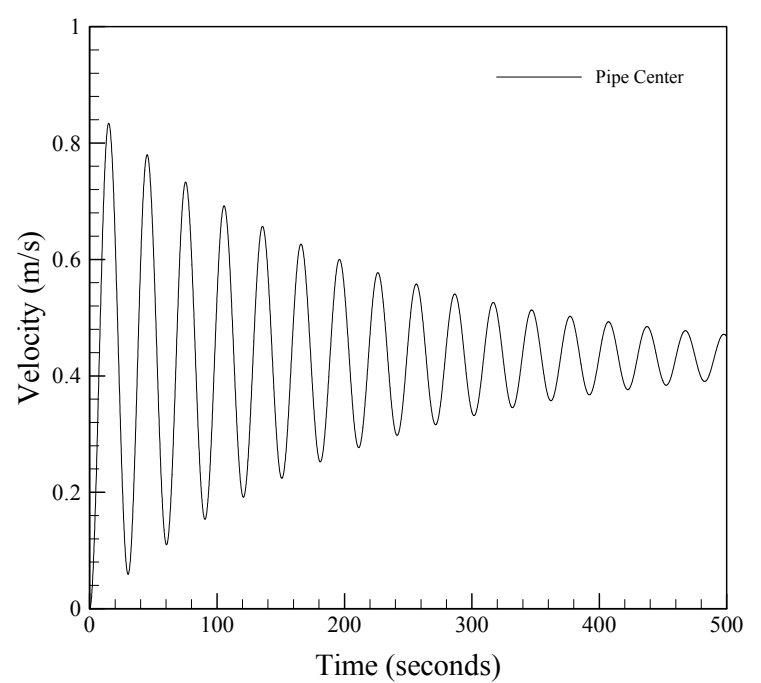

Fig. 7: Variation of velocity at the center of the pipe

The computed results show that the maximum water surface level is about 32.5 times higher than the final steady state water surface level, while the minimum velocity that occurs after the first peak is about $13.7 \%$ of the final steady state velocity. To improve the operating conditions, i.e., reduce the water surface oscillation and increase the minimum velocity magnitude occurring after the first peak, two options are available. The first option is to increase the surface area of the upstream reservoir, which may prove costly for the existing water treatment plants and even for new reservoirs. The second option is to increase the flow rate slowly to the final steady state inflow rate. However, the time span of flow increase and the manner in which the flow increase is achieved are open to selection.

In this study, a linear increase in discharge from zero to the prescribed discharge for different time span is analyzed. The results are shown in Figs. 8 and 9. Fig. 8 shows an exponential type decay of the maximum water surface level in the upstream reservoir with increase in time over which the linear increase in the discharge takes place. For example, if the flow increase from zero to the prescribed value is achieved in 40 seconds, the maximum water surface level above the initial level is reduced to $9.4 \mathrm{~cm}$ compared to $39 \mathrm{~cm}$ for the original case. The figure also shows that a major reduction in the maximum water surface level is achieved in the first 40 seconds. Fig. 9 shows the variation of minimum velocity at the center of the pipe 
with change in flow increase time. The minimum velocity reported is the one achieved after the first peak in the velocity. The figure shows that if the flow increase is achieved in 40 seconds, the minimum velocity in the system after the first peak is $36 \mathrm{~cm} / \mathrm{s}$ and is $83 \%$ of the final steady state velocity. Thus a flow increase time of 40 seconds is adequate for the present study. It should be mentioned that the results are valid for the set up discussed in this work, however, analysis can be carried out for different configuration by adapting the present methodology.

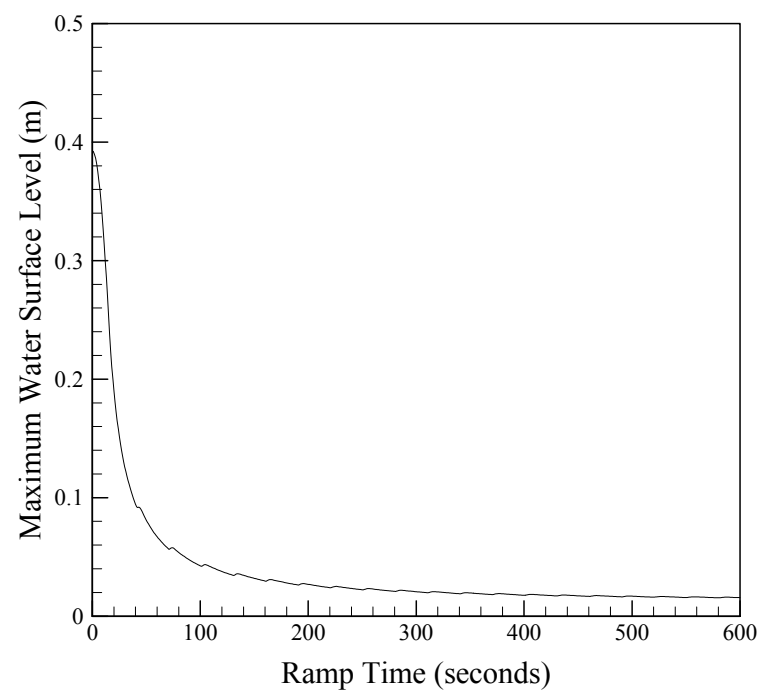

Fig. 8: Maximum water surface level in upstream reservoir

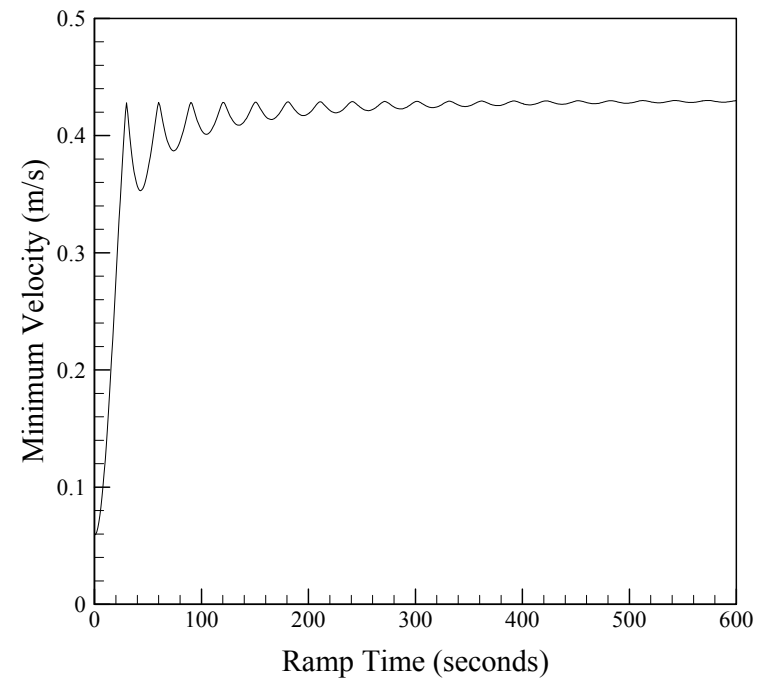

Fig. 9: Minimum velocity at the middle of the pipe

\section{CONCLUSIONS}

The full mass and momentum conservation equations based on the elastic theory are solved using the method of characteristics for predicting the water surface and velocity oscillations, for a two-reservoir system connected by a pipe, due to the start of a through flow. Appropriate boundary conditions are devised for obtaining the solution. As a first step, the solution obtained using the elastic theory is compared to the classical solution for the variation of the water surface elevation in a U-tube resulting from an initial forced difference in the water level. The method is then used to predict the water surface level and velocity oscillations in an existing system due to the start of a through flow. The results show that the maximum water surface level in the upstream reservoir is about 32.5 times the final steady state water surface level and the minimum velocity after the first peak velocity is $13.7 \%$ of the final steady state velocity.

In order to improve the operating conditions, a linear increase in discharge from zero to the prescribed value is investigated. The results show that a significant reduction in maximum water surface level and increase in minimum velocity after the first peak velocity can be obtained if the discharge is increased linearly to the design discharge in 40 seconds. The minimum velocity is increased from $13.6 \%$ to $85 \%$ of the final steady state velocity and the maximum water surface level is reduced from $39 \mathrm{~cm}$ to $9.4 \mathrm{~cm}$ above the initial water level. Although the results obtained are valid only for the system analyzed in this study, the methodology developed can be adopted effectively for other systems both for analysis and rectification.

\section{REFERENCES}

1. Haaland, S.E., 1983. Simple and explicit formulas for friction factor in turbulent pipe flow. Journal of Fluids Engineering Science, 20: 1073-1078.

2. Halliwell, A.R., 1963. Velocity of a water-hammer wave in an elastic pipe. ASCE Journal of the Hydraulics Division, 89: 1-21.

3. Streeter, V.L., 1962. Fluid Mechanics. Third Edition, McGraw-Hill, New York, pp: 555.

4. Streeter, V.L., and E.B. Wylie and K.W. Bedford, 1998. Fluid Mechanics. $9^{\text {th }}$ Edition, McGraw-Hill, New York, New York, pp: 740.

5. Watters, G.Z., 1984. Analysis and Control of Unsteady Flow in Pipelines. $2^{\text {nd }}$ Edition, Butterworth, Boston, pp: 349. 